\title{
Rede social na Internet como apoio à Formação Docente
}

\author{
Gilmara T. Barcelos ${ }^{1}$, Silvia Cristina F. Batista ${ }^{1}$ \\ ${ }^{1}$ Instituto Federal Fluminense Campus Campos-Centro \\ Rua Dr. Siqueira, 273. Parque Dom Bosco. CEP: 28030-130. Campos dos \\ Goytacazes/RJ \\ gilmarabeiff.edu.br, silviaceiff.edu.br
}

\begin{abstract}
This paper analyzes a case study carried in a subject given in a lato sensu post-graduation course for teachers. The subject program promoted the discussion of the use of digital technologies (DT), and used an Internet social network (ISN) to support the activities. Thus, this article aims to analyze difficulties and advantages of the use of ISN in teacher training programs. The study starts with a reflection of the importance of pedagogical use of ISN. Following, the case study is contextualized, and the methodological procedures are described. The paper closes with a discussion of the difficulties and advantages of using ISN based on data collected. In general, the use of the ISN was considered a positive one.
\end{abstract}

Resumo. Este artigo analisa um estudo de caso promovido em uma disciplina de um curso de especialização para professores. Nessa disciplina, discutiu-se o uso pedagógico de tecnologias digitais (TD) e, apoiando as atividades, foi utilizada uma rede social na Internet (RSI). Este artigo visa, então, analisar dificuldades e vantagens do uso de uma RSI na preparação de professores. Para tanto, inicialmente reflete-se sobre a importância do uso pedagógico de RSI. A seguir, é descrito o contexto do estudo de caso e os procedimentos metodológicos adotados. Finalizando, são analisadas vantagens e dificuldades do uso da RSI, levantadas por meio de observação, questionário e registros dos usuários na rede. De maneira geral, o uso da RSI foi considerado positivo.

\section{Introdução}

O surgimento da Internet e, em especial, das funcionalidades da Web 2.0 ${ }^{1}$ possibilitaram a criação de espaços de troca virtuais. Nesse contexto, com o suporte dos recursos Web 2.0, as redes sociais ampliaram seus horizontes. Tais recursos possibilitaram uma nova forma de relacionamento, independente de tempo e espaço, por meio das chamadas redes sociais na Internet (RSI). Estas redes permitiram que, os até então consumidores, tornassem-se produtores, criando e compartilhando informações [Attwell 2007].

Assim, diversas pesquisas estão sendo promovidas, investigando o uso de RSI para fins pedagógicos [Tairi et al. 2008; Moreira e Monteiro 2010; Paião 2010; Moran, Seaman e Tinti-Kane 2011; Abreu et al. 2011]. Porém, trata-se de uma área de pesquisa ainda recente e, portanto, investigações relativas ao assunto são fundamentais.

Independente das RSI, as tecnologias digitais (TD) associadas à Internet possibilitam experimentação de papéis sociais, ampliação das relações interpessoais e do acesso à informação [Moço 2010], entre outras ações. Muitos professores

\footnotetext{
${ }^{1}$ Os princípios fundamentais da Web 2.0 são: uso da Web como plataforma e aprimoramento de serviços, a partir do aumento do número de usuários [O’Reilly 2005].
} 
desconhecem essas possibilidades, fato que, em alguns casos, inibe a experimentação [Moço 2010]. Porém, é importante mudar esse contexto, ou seja, professores e também os pais precisam conhecer, experimentar, investigar as TD para que possam ajudar os jovens a construir uma relação saudável com as referidas tecnologias [Moço 2010].

Nesse contexto, foi realizado um estudo de caso, com abordagem de análise dos dados, predominantemente, qualitativa. $\mathrm{O}$ referido estudo ocorreu em uma disciplina ${ }^{2}$ ministrada em uma pós-graduação lato sensu, destinada a professores da Educação Básica, em uma Instituição Federal. A disciplina foi ministrada no período de março a maio de 2012, tendo como foco o uso pedagógico de Tecnologias Digitais (TD). Apoiando as atividades, foi utilizada uma RSI, implementada por meio da plataforma Elgg. O uso da referida RSI foi observado ao longo da disciplina, visando levantar dificuldades e pontos favoráveis associados ao mesmo. Além de observação, a coleta de dados também foi realizada por meio de questionário e registros da própria rede.

Este artigo visa, então, analisar as dificuldades e vantagens do uso da RSI, identificadas no estudo de caso. Para tanto, na Seção 2, discute-se a importância do uso de RSI na educação. A seguir, na Seção 3, é descrito o contexto do estudo de caso e os procedimentos metodológicos adotados. $\mathrm{Na}$ Seção 4, são analisadas vantagens e dificuldades do uso da RSI, por meio dos dados levantados. Finalizando, na Seção 5, são apresentadas considerações sobre o estudo promovido.

\section{Redes sociais na Internet}

No âmbito da preparação de professores para uso pedagógico das TD, considera-se que as redes sociais e os diversos tipos de comunidades na Internet são importantes. Uma rede social é definida como um conjunto de dois elementos: atores, ou seja, nós (pessoas, instituições ou grupos) e suas conexões-interações ou laços entre os nós [Recuero 2009]. Araújo e Assis (2011) destacam quatro aspectos que caracterizam as redes: i) sustentam-se pela vontade e afinidade de seus integrantes, que são os responsáveis pelo alcance do objetivo; ii) não são lineares, estendem-se em várias direções; iii) possuem como propriedade organizacional característica a horizontalidade, ou seja, a estrutura das redes é uma alternativa à forma de organização piramidal; iv) são multidimensionais, remetem para as muitas dimensões (níveis, camadas, círculos).

As formas de expressão numa rede social concretizam-se no estabelecimento de interações entre os nós da rede (atores da rede). Essas interações têm um caráter dinâmico e a análise das mesmas permite compreender que tipos de relacionamentos emergem na rede e que se constituem em laços sociais. Os laços podem ser fortes ou fracos de acordo com a qualidade das interações e das trocas sociais realizadas entre os atores. Laços fortes são aqueles que se caracterizam pela intimidade e pela proximidade, já os fracos caracterizam-se por relações esparsas, que não traduzem proximidade e intimidade [Granovetter 2000]. Dessa forma, os laços fortes constituem as redes menos instáveis. As trocas que ocorrem nas redes sociais geram elemento imaterial construído e negociado entre os atores e que permite o aprofundamento dos laços e a sedimentação dos grupos [Recuero 2009]. Segundo Putnam (1993), capital social refere-se à conexão entre indivíduos, são normas e valores que governam interações entre as pessoas. $\mathrm{O}$ "social" do capital social ressalta que esses recursos não são bens pessoais, e que

\footnotetext{
${ }^{2} \mathrm{O}$ objetivo geral dessa disciplina é contribuir para o uso pedagógico de tecnologias digitais na prática docente, visando à construção de conhecimentos.
} 
residem em redes sociais.

Para a criação de RSI, faz-se necessário o uso de plataformas como, por exemplo: Orkut, Facebook, SocialGO, Meezoog, WackWall, Elgg, entre outras. Essas plataformas não são as RSI, mas as representam [Recuero 2009], as redes em si são criadas a partir das interações, dos laços sociais estabelecidos e do capital social gerado. Essas não são pré-construídas pelas plataformas, e, sim, apropriadas pelos atores sociais que lhes conferem sentido e que as adaptam as suas práticas sociais [Recuero 2012].

As redes sociais, por meio das interações, vêm modificando diversas áreas da atividade humana, a saber: comércio, indústria, economia, artes, cultura e educação, desde os mais remotos tempos da humanidade [Clark e Roberts 2010]. Geralmente, uma RSI é usada para conhecer pessoas, disponibilizar fotos, vídeos, comentários, comercializar produto. Porém, também podem representar um importante recurso de apoio às atividades educacionais [Clark e Roberts 2010].

Pesquisas recentes vêm mostrando que o uso pedagógico de RSI pode representar uma estratégia bastante interessante [Paião 2010]. Em particular, o estudo realizado por Moreira e Monteiro (2010) sinalizou que a criação de espaços virtuais complementares à aprendizagem presencial é importante para a promoção e reforço das interações professor/aluno e alunos/alunos, na partilha de conhecimentos e nas estratégias de trabalho cooperativo. Defende-se, inclusive, que estas redes podem auxiliar programas de formação continuada de professores, possibilitando que estes vivenciem as vantagens das funcionalidades das RSI e assim tenham confiança para usálas com seus alunos [Barcelos, Passerino e Behar 2010].

Goldfarb et al. (2011) listam benefícios no uso pedagógico de RSI, apontados na literatura, tais como: i) identificação precoce de necessidades dos estudantes e avaliação formativa; ii) estabelecimento de comunidades que compartilham idéias, abordagens e recursos; iii) engajamento dos alunos, pelo fato dos mesmos estarem bem familiarizados com as RSI; iv) melhoria de desempenhos dos alunos, propiciada pelo maior envolvimento nas discussões promovidas em aula; v) gestão da informação, favorecida pela capacidade das RSI integrarem várias ferramentas.

Pesquisa realizada com educadores (professores, diretores e bibliotecários) dos EUA [edWeb.net et al. 2010], levantou uma visão das práticas e políticas do uso pedagógico de RSI. Com base nos dados obtidos, os autores apresentaram recomendações sobre a questão: i) diretores e professores precisam adquirir maior experiência de uso educacional de RSI, possibilitando melhor compreensão das possibilidades abertas pelas mesmas; ii) práticas promissoras devem ser divulgadas, compartilhando formas de integrar uma RSI ao currículo; iii) políticas de uso de sites que tenham características sociais/colaborativas devem ser estabelecidas.

Outra pesquisa realizada nos EUA [Moran, Seaman e Tinti-Kane 2011], com 1920 professores do ensino superior, sinalizou que a maneira que os professores usam as RSI em sala de aula ainda é muito passiva. Destacam-se alguns resultados dessa pesquisa: i) em sala de aula, os principais usos identificados foram assistir vídeos e ouvir podcast; ii) não foram identificadas evidências de que docentes que atuam on-line tenham maior nível de consciência no uso de RSI; iii) 58\% dos participantes da pesquisa concordam que o uso de RSI pode ser valioso para aprendizagem colaborativa [Moran, Seaman e Tinti-Kane 2011]. É importante que professor perceba as RSI como meio e 
possibilidade de ampliar os espaços educacionais, descentralizar o acesso ao saber, mudar a lógica de comunicação e aproximar professores e alunos.

No Brasil, de acordo com José Armando Valente [Klix 2011], o uso pedagógico das RSI tem ocorrido em iniciativas pontuais como, por exemplo, o uso de blogs e outros recursos em aula, mas, geralmente por interesse particular de alguns profissionais. Além disso, o autor destaca que, em geral, o uso é para divulgar algum conteúdo que não tenha sido possível apresentar em aula e para receber material de aluno. Para Valente [Klix 2011], essas ações não são inovadoras, apenas contribuem para transmissão de informação. Não se questiona o valor desses usos, mas considera-se que é possível ir além, como no estudo de caso descrito nas seções seguintes.

\section{Estudo de Caso: Contexto e procedimentos metodológicos}

O estudo de caso descrito foi promovido em uma disciplina ministrada em uma PósGraduação lato sensu para professores da Educação Básica, promovida por uma Instituição Federal. A carga horária da referida disciplina foi de 32 horas, no período de 16/03 a 11/05/2012. A mesma foi ministrada de forma conjunta pelas autoras deste artigo, visando atingir os seguintes objetivos: i) analisar o uso pedagógico das TD na educação; ii) discutir políticas públicas direcionadas à implementação e utilização dessas tecnologias nas escolas; iii) distinguir diferentes abordagens do uso de softwares educacionais no processo de ensino e aprendizagem; iv) avaliar criticamente, diferentes softwares e sites educacionais, de acordo com diferentes abordagens; v) analisar o uso da Internet como tecnologia para construção de conhecimentos.

Todos os 27 alunos da disciplina eram professores do Ensino Fundamental e Médio, em áreas diversas, alguns já atuando efetivamente, outros ainda não. Do total de alunos, 23 (aproximadamente, 85\%) eram do sexo feminino. A categorização dos alunos por faixa etária pode ser observada na tabela 1.

Tabela 1. Faixa Etária dos Alunos

\begin{tabular}{|l|c|c|c|c|c|c|}
\hline Faixa Etária & $21-26$ & $27-32$ & $33-38$ & $39-44$ & $45-50$ & Acima de 50 \\
\hline Alunos (\%) & 29,63 & 33,33 & 18,53 & 14,81 & 3,70 & 0 \\
\hline
\end{tabular}

Observa-se que a faixa etária estava concentrada entre 21 e 32 anos, mas também havia dez professores mais experientes, entre 33 e 50 anos.

Para a referida Pós-Graduação, foi implementada uma rede social por meio da plataforma Elgg. Essa RSI foi utilizada na disciplina descrita neste artigo, apoiando as atividades propostas e expandindo os limites da sala de aula. A escolha da plataforma Elgg fundamentou-se nos seguintes quesitos: gratuidade, idioma (Português), variedade de ferramentas e facilidade no uso dos recursos disponibilizados. Essa plataforma permite que usuários publiquem, organizem e compartilhem materiais de trabalho e de suporte a aprendizagem. Cada usuário tem a possibilidade de escolher quais recursos serão mostrados para quais usuários. A plataforma oferece diversas ferramentas de gestão do conhecimento, como blog, microblog, ferramentas de colaboração e de comunicação. Além disso, permite um grande controle dos recursos tanto por parte do administrador como dos demais usuários. Alguns estudos sobre o uso da plataforma Elgg no contexto educacional já foram realizados [Tairi et al. 2008; Ryberg 2008; Dias, Oliveira e Alves 2009], porém, não foi identificado nenhum estudo utilizando essa plataforma na formação continuada de professores. 
É importante ressaltar que a plataforma Elgg pode ser considerada como um Personal Learning Environments (PLE), entendendo-a como um conjunto de ferramentas integradas, com grande controle por parte do usuário [Mota 2009]. Os PLE representam para a educação, os princípios de e-Learning 2.0, do poder e autonomia do usuário, da abertura, da colaboração e da partilha [Attwell 2007; Mota 2009]. Um PLE, por definição, apresenta uma proposta mais inovadora do que um ambiente virtual de aprendizagem. Esses últimos ainda estão próximos da metáfora da sala de aula e neles os alunos vivenciam a mesma experiência, veem o mesmo conteúdo, organizado da mesma forma, com as mesma ferramentas [Wilson et al. 2006].

O uso da rede social foi observado ao longo da disciplina, visando levantar as dificuldades e os pontos favoráveis associados ao mesmo. Para a coleta e análise de dados, adotou-se uma proposta mista de investigação, com abordagens qualitativas e quantitativas. No entanto, destaca-se que, na análise quantitativa promovida, foram utilizadas apenas técnicas da Estatística Descritiva, que engloba um conjunto de métodos destinados à organização e descrição de dados.

A coleta de dados foi realizada por meio de observação, questionário e registros da rede social. Com relação à observação, adotou-se o tipo não-estruturada, entendendose que a mesma permitiria captar situações imprevistas, tão comuns no contexto educacional. Como afirmam Laville e Dione (1999), observações estruturadas são delimitadas e podem levar o pesquisador a uma visão parcial ou superficial da situação. Com relação ao questionário, o mesmo foi composto por três questões relacionadas ao uso de redes sociais de maneira geral e oito questões sobre o uso da rede social da PósGraduação. Dessas oito questões, uma era aberta, solicitando comentários relacionados às funcionalidades da rede ou à participação do aluno na mesma. Os registros dos alunos na rede social foram acompanhados por meio das diversas ferramentas existentes na própria plataforma.

Segundo Creswell (2007), é importante tentar garantir a consistência dos dados e, nesse sentido, uma estratégia é a triangulação de diferentes fontes. Assim, ao utilizar as três técnicas de coleta citadas, visou-se promover essa triangulação, buscando maior consistência dos dados.

\section{Rede social na Internet: vantagens e desvantagens}

Ao longo da disciplina, a RSI foi utilizada tanto no apoio às atividades da disciplina, quanto como meio de comunicação entre as professoras e os alunos e, também, entres eles. Inicialmente, os alunos manifestaram dificuldades em relação aos fóruns de discussão, no que diz respeito a postagens de arquivos e disponibilização de links dos mesmos. Com o tempo, foi possível observar que o uso da rede social, para as finalidades propostas, foi se tornando mais natural. Porém, ainda assim, algumas pessoas demonstravam dificuldades de identificação do local de postagens de determinadas atividades, deixando perceber que as mesmas se sentiam meio desorientadas com a estrutura não linear da rede. No entanto, era perceptível que a maioria se sentia confortável usando uma RSI para fins educacionais, demonstrando, até mesmo, entusiasmo por estar usando um recurso atual em sua formação.

Os dados identificados no conteúdo da própria rede também foram analisados. Foi possível observar que os comentários e reflexões dos alunos nos fóruns eram de excelente qualidade, com posicionamentos bem fundamentados. Isso sinaliza que os 
problemas iniciais com os fóruns eram técnicos e não decorrentes de dificuldade de argumentação. $\mathrm{O}$ uso do microblog por parte dos alunos também merece ser destacado. O mesmo foi muito utilizado para: i) trocar informações com os professores e colegas; ii) divulgar vídeos, sites e eventos relacionados à Informática na Educação; iii) expressar sentimentos e compartilhar novidades. A troca de emails, por meio de recurso da RSI, entre alunos e professoras também foi significativo. As professoras, muitas vezes, usavam o email para solicitar correções em trabalhos, de forma mais pessoal. Os alunos, em geral, enviavam email para avisar sobre faltas às aulas e respectivos motivos.

Ao final da disciplina foram levantados outros dados por meio de um questionário respondido pelos 27 alunos. Tais dados são discutidos a seguir. Com relação ao uso de redes sociais antes da Pós-Graduação, a tabela 2 mostra os resultados.

Tabela 2. Uso de Redes Sociais Antes da Pós-Graduação

\begin{tabular}{|c|c|c|}
\hline Opções & Sim & Não \\
\hline Alunos (\%) & 74,07 & 25,93 \\
\hline
\end{tabular}

Os 20 alunos que já utilizavam redes sociais foram questionados, ainda, sobre a finalidade de uso das mesmas. Era possível assinalar opções múltiplas nessa questão. São apresentadas as finalidades mais destacadas pelos alunos: i) compartilhar comentários $(90 \%)$; ii) fazer amigos $(65 \%)$; iii) compartilhar fotos $(65 \%)$; iv) trocar experiências profissionais com pessoas de mesma área (60\%); v) interagir com alunos e colegas de profissão (55\%). Os dois últimos itens merecem destaque, por sinalizarem um uso educacional de redes sociais.

As questões seguintes são diretamente relacionadas à RSI adotada na PósGraduação. São sete afirmativas, diante das quais cada aluno deveria se posicionar em uma das opções dadas: Concordo Plenamente, Concordo, Não Concordo Nem Discordo, Discordo, Discordo Plenamente. Em cada uma das tabelas abaixo, $100 \%$ dos participantes correspondem a 27 alunos.

A primeira afirmativa proposta foi "Participar dos fóruns de discussão da rede social foi fácil". A Tabela 3 apresenta os dados levantados.

Tabela 3. Facilidade de Participação nos Fóruns de Discussão

\begin{tabular}{|c|c|c|c|c|c|}
\hline Opções & $\begin{array}{c}\text { Concordo } \\
\text { plenamente }\end{array}$ & Concordo & $\begin{array}{c}\text { Não concordo } \\
\text { nem discordo }\end{array}$ & Discordo & $\begin{array}{c}\text { Discordo } \\
\text { plenamente }\end{array}$ \\
\hline Alunos (\%) & 33,33 & 29,64 & 33,33 & 3,70 & 0 \\
\hline
\end{tabular}

Os percentuais da tabela 3 estão de acordo com a realidade observada durante a disciplina. Com o tempo, as dificuldades de alguns alunos foram diminuindo, mas não a ponto de que os mesmos considerassem fácil a participação nos fóruns. Portanto, o percentual na opção "Não concordo nem discordo" é coerente. Conjuntamente, as opções "Concordo plenamente" e "Concordo" atingiram 62,97\%, o que sinaliza que, para a maioria, a participação foi fácil. No entanto, somente 33,33\% concordaram plenamente com a afirmativa, o que parece indicar que, no geral, a participação foi fácil, mas não muito fácil. Esses índices podem ser decorrentes do fato da ferramenta fórum ser nova para a maioria dos professores, aspecto observado durante as aulas.

Com relação à afirmativa "De maneira geral, foi fácil identificar os locais de postagens das atividades", a tabela 4 mostra os resultados obtidos. 
Tabela 4. Facilidade de Identificação do Local de Postagens das Atividades

\begin{tabular}{|c|c|c|c|c|c|}
\hline Opções & $\begin{array}{c}\text { Concordo } \\
\text { plenamente }\end{array}$ & Concordo & $\begin{array}{c}\text { Não concordo } \\
\text { nem discordo }\end{array}$ & Discordo & $\begin{array}{c}\text { Discordo } \\
\text { plenamente }\end{array}$ \\
\hline Alunos (\%) & 14,81 & 48,15 & 11,11 & 18,52 & 7,41 \\
\hline
\end{tabular}

Novamente, os percentuais da tabela 4 são coerentes com as dificuldades observadas. Embora para a maioria $(62,96 \%)$, a identificação tenha sido fácil, sete pessoas $(25,93 \%)$ discordaram disso, e três $(11,11 \%)$ mantiveram-se neutras. Cabe lembrar, que 25,93\% dos alunos não utilizavam redes sociais, antes da Pós-Graduação. Como mencionado anteriormente, a estrutura não linear da RSI pode provocar certa desorientação em usuários menos habituados. A postagem de atividades em locais diferentes dos indicados pelas professoras, por parte de alguns alunos, retrata bem a dificuldade dos mesmos.

Os dados relativos à afirmativa "As ferramentas que a rede social apresenta são satisfatórias" são mostrados na tabela 5 .

Tabela 5. Satisfação com as ferramentas da rede social

\begin{tabular}{|c|c|c|c|c|c|}
\hline Opções & $\begin{array}{c}\text { Concordo } \\
\text { plenamente }\end{array}$ & Concordo & $\begin{array}{c}\text { Não concordo } \\
\text { nem discordo }\end{array}$ & Discordo & $\begin{array}{c}\text { Discordo } \\
\text { plenamente }\end{array}$ \\
\hline Alunos (\%) & 33,33 & 44,45 & 11,11 & 11,11 & 0 \\
\hline
\end{tabular}

Conjuntamente, as opções "Concordo plenamente" e "Concordo" totalizam $77,78 \%$, assim, para a maioria, as ferramentas da rede social foram satisfatórias. Porém, o percentual específico da opção "Concordo plenamente" não foi alto, o que parece indicar que, em geral, essas ferramentas ainda podem evoluir em termos educacionais.

A tabela 6 mostra os percentuais relativos à afirmativa "A utilização da rede social contribuiu para o desenvolvimento das atividades da disciplina".

Tabela 6. Contribuição da Rede Social para as Atividades da Disciplina

\begin{tabular}{|c|c|c|c|c|c|}
\hline Opções & $\begin{array}{c}\text { Concordo } \\
\text { plenamente }\end{array}$ & Concordo & $\begin{array}{c}\text { Não concordo } \\
\text { nem discordo }\end{array}$ & Discordo & $\begin{array}{c}\text { Discordo } \\
\text { plenamente }\end{array}$ \\
\hline Alunos (\%) & 59,26 & 18,52 & 11,11 & 3,70 & 7,41 \\
\hline
\end{tabular}

O percentual da opção "Concordo plenamente" indica boa aceitação em relação à afirmativa apresentada. Considerando-se, conjuntamente, as opções "Concordo plenamente" e "Concordo", o percentual de concordância atinge 77,78\%, que foi considerado um percentual coerente com o que foi observado. Os demais percentuais podem ser justificados pelas dificuldades técnicas apresentadas por alguns alunos e, também, pela falta de prática do uso de recursos tecnológicos no apoio à educação.

Os resultados relativos à afirmativa "A utilização da rede social contribuiu para a interação com as professoras e com os colegas" são apresentados na tabela 7.

Tabela 7. Contribuição da Rede Social para a Interação com Professoras e Colegas

\begin{tabular}{|c|c|c|c|c|c|}
\hline Opções & $\begin{array}{c}\text { Concordo } \\
\text { plenamente }\end{array}$ & Concordo & $\begin{array}{c}\text { Não concordo } \\
\text { nem discordo }\end{array}$ & Discordo & $\begin{array}{c}\text { Discordo } \\
\text { plenamente }\end{array}$ \\
\hline Alunos (\%) & 55,56 & 29,63 & 3,70 & 3,70 & 7,41 \\
\hline
\end{tabular}

O percentual de concordância de $85,19 \%$ indica que a rede social foi muito importante para a interação entre os integrantes da disciplina. Os demais percentuais 
foram considerados pertinentes ao contexto considerado. Esses dados estão de acordo com a pesquisa promovida por Moreira e Monteiro (2010) que também sinalizou que espaços virtuais como as RSI, apoiando a aprendizagem presencial, são importantes para interações entre professor/aluno e alunos/alunos.

A tabela 8 mostra os resultados relativos à afirmativa "A possibilidade de compartilhar informações diversas, por meio de arquivos, links, blogs, microblog na rede social foi importante para o processo de ensino e aprendizagem".

Tabela 8. Importância do Compartilhamento de Informações

\begin{tabular}{|c|c|c|c|c|c|}
\hline Opções & $\begin{array}{c}\text { Concordo } \\
\text { plenamente }\end{array}$ & Concordo & $\begin{array}{c}\text { Não concordo } \\
\text { nem discordo }\end{array}$ & Discordo & $\begin{array}{c}\text { Discordo } \\
\text { plenamente }\end{array}$ \\
\hline Alunos (\%) & 48,15 & 37,04 & 3,70 & 11,11 & 0 \\
\hline
\end{tabular}

Embora o percentual na opção "Concordo plenamente" tenha sido menor do que na tabela 7, o percentual total de concordância foi, novamente, de $85,19 \%$, o que indica que o compartilhamento de informação contribuiu para o processo de ensino e aprendizagem. A gestão de informações, favorecida pela capacidade de integração de várias ferramentas das RSI também foi apontada Goldfarb et al. (2011), como um ponto positivo do uso pedagógico dessas redes.

Finalizando, a tabela 9 mostra os dados relativos à afirmativa "As funcionalidades da rede social possibilitaram que a relação entre alunos e professores fosse menos hierárquica".

Tabela 9. Redução da Hierarquia na Relação Professsor-Aluno

\begin{tabular}{|c|c|c|c|c|c|}
\hline Opções & $\begin{array}{c}\text { Concordo } \\
\text { plenamente }\end{array}$ & Concordo & $\begin{array}{c}\text { Não concordo } \\
\text { nem discordo }\end{array}$ & Discordo & $\begin{array}{c}\text { Discordo } \\
\text { plenamente }\end{array}$ \\
\hline Alunos (\%) & 44,44 & 37,04 & 7,41 & 3,70 & 7,41 \\
\hline
\end{tabular}

Observa-se que, para a maioria dos alunos, as funcionalidades da rede contribuíram para reduzir a hierarquia na relação professor-aluno. A possibilidade de estabelecer uma comunidade, entre professores e alunos, compartilhando informações e recursos foi destacada por Goldfarb et al. (2011). Segundo os autores, as RSI são ambientes encorajadores e intrinsecamente democráticos.

As respostas da questão aberta, sobre o uso da rede social, permitiram observar que, de maneira geral, a proposta de utilização de uma RSI para fins educacionais foi bem aceita pelos alunos. Alguns comentários, como os dois apresentados a seguir, mostraram que as dificuldades iniciais foram sendo superadas ao longo da disciplina.

\footnotetext{
Pra mim a nossa rede foi um desafio. Primeiramente eu era avessa a esse tipo de ambiente, não por não gostar, mas por medo de não saber manusear ou interagir. Mas depois percebi que minhas dificuldades e duvidas foram sanadas (Aluno F);

Embora tivesse pouquíssimo conhecimento e domínio acerca do uso de rede social, pude perceber que, no contexto que estamos inseridos, é de suma importância para efeito comunicativo e de interação, além do fator principal que é utilizar esses recursos na prática educacional [...](Aluno $\mathrm{M})$.
}

De maneira geral, os resultados foram positivos e, como recomendado por edWeb.net et al. (2010), é importante divulgar experiências promissoras de uso pedagógico de RSI. Em resumo, foi possível destacar que o uso de RSI foi importante 
para: i) o desenvolvimento das atividades da disciplina; ii) a interação com as professoras e colegas; iii) o compartilhamento de informações por meio das ferramentas da rede social; iv) redução da hierarquia na relação professsor-aluno; v) o alcance do objetivo geral da disciplina. Como dificuldade, foi possível destacar: i) a identificação dos locais de postagem das atividades; ii) postagem de arquivos e links nos fóruns.

\section{Considerações Finais}

As RSI têm potencial para colaborar com a educação, como foi evidenciado no estudo de caso relatado. No âmbito da formação de professores para uso pedagógico das $\mathrm{TD}$, considera-se que a utilização das mesmas possibilita ao professor, além dos benefícios gerais, a oportunidade de conhecer, como aluno, um recurso que poderá aplicar em suas próprias turmas.

A análise de todos os instrumentos de coleta de dados possibilitou afirmar que o uso da rede foi uma estratégia positiva para o desenvolvimento e para a proposta da disciplina. Como mencionado, dificuldades ocorreram, porém, é possível que as mesmas tenham também sofrido influência da pouca habilidade de uso do computador, demonstrada por alguns alunos. Cabe ressaltar que, embora alguns tenham manifestado dificuldades com relação à participação nos fóruns, a qualidade das discussões promovidas foi excelente.

Finalizando, é importante mencionar que a utilização da plataforma Elgg, nos moldes da experiência relatada, exige instalação em servidor e manutenção técnica, o que requer, em geral, apoio institucional. Trata-se de um aspecto importante a ser considerado, pois nem toda instituição educacional tem possibilidade/interesse em oferecer esse apoio.

\section{Referências}

Abreu, J., Claudeivan, L., Veloso, F. e Gomes, A. S. (2011). Análise das Práticas de Colaboração e Comunicação: Estudo de Caso utilizando a Rede Social Educativa Redu. In: Simpósio Brasileiro de Informática na Educação (SBIE), 22 - Workshop de Informática na Escola (WIE), 17, Aracaju, SE, SBC, p. 1246-1255.

Araújo, F. O. e Assis, V. B. G. de (2011). Redes Sociais na sala de aula: uma nova perspectiva para o sucesso profissional. Niterói, RJ: Editora da UFF.

Attwell, G. (2007). Personal Learning Environments - the future of eLearning? Elearningpaper, v.2, n. 1, Jan.

Barcelos, G. T., Passerino, L. M. e Behar, P. A. (2010) Análise dos Impactos da Integração de Tecnologias na Formação Inicial de Professores de Matemática sobre a prática docente: um estudo de caso. In: Workshop de Informática na Escola (WIE), 15, Belo Horizonte, MG, SBC.

Clark, L. A. e Roberts, S. J. (2010). Employer's Use of Social Networking Sites: A Socially Irresponsible Practice. Journal of Business Ethics, 95, 507-525.

Creswell, J. W. (2007). Projeto de Pesquisa: métodos qualitativo, quantitativo e misto. Tradução de Luciana de Oliveira da Rocha. 2 ed. Porto Alegre: Artmed, 2007.

Dias, C. M. V., Oliveira, L. R. M. e Alves, M. P. C. (2009). Recognition, Validation and Certification of Competences Using Eportfolio. Contributions to Changing the Evaluation Paradigm and to the Development of Computer Science Literacy. In: World Conference on E-Learning in Corporate, Government, Healthcare, and Higher 
Education, Chesapeake, VA, AACE, p. 2535-2538.

edWeb.net, IESD, Inc., MCH, Inc. e MMS Education (2010). "School Principals and Social Networking in Education: Practices, Policies, and Realities in 2010". http://www.edweb.net/fimages/op/PrincipalsandSocialNetworkingReport.pdf, julho, 2012.

Granovetter, M. (2000). La fuerza de los vínculos débiles. Política y Sociedad, n. 33. Madrid, p. 41-56.

Goldfarb, A., Pregibon, N., Shrem, J. e Zyko, E. (2011). Informational Brief on Social Networking in Education. http://www.p12.nysed.gov/technology/internet_safety/documents/InformationalBriefo nSocialNetworkinginEducation.pdf, julho, 2012.

Klix, T. (2011). Educador quer Redes Sociais no Currículo Escolar. Último Segundo Educação.http://ultimosegundo.ig.com.br/educacao/educador\%20quer\%20redes\%20s ociais\%20no\%20curriculo\%20escolar/n1238187320827.html\%20, julho, 2012.

Laville, C. e Dionne, J. (1999). A Construção do Saber: manual da metodologia da pesquisa em ciências humanas. Porto Alegre, RS, Brazil: Artmed.

Moço, A. (2010). Um desafio real. Revista nova escola, n. 236, p. 92-96, Out. 2010.

Moran, M., Seaman J. e Tinti-Kane, H. (2011). Teaching, Learning, and Sharing: How Today's Higher Education Faculty Use Social Media. Boston, USA: Pearson Learning Solutions.

Moreira, J. A. M. e Monteiro, A. A. (2010). O trabalho pedagógico em cenários presenciais e virtuais no ensino superior. Educação, Formação \& Tecnologias, v.3, n. 2, p. 82-94.

Mota, J. (2009). Personal Learning Environments: contributos para uma discussão do conceito. Educação, Formação \& Tecnologias, v.2, n.2, pp. 5-21, Nov. de 2009.

O'Reilly, T. (2005). What is Web 2.0 - Design Patterns and Business Models for the Next Generation of Software. O'Reilly Publishing.

Paião, C. (2010). Plataformas sociais auxiliam a construção do conhecimento? Com Ciência: revista eletrônica de jornalismo científico, 121.

Putnam, R. (1993). Making democracy work: civic traditions in modem Italy. Princeton: Princeton University Press.

Recuero, R. (2009). Redes sociais na Internet. Porto Alegre, RS: Sulina.

Recuero, R. (2012) A conversação em rede: comunicação mediada pelo computador e redes sociais na internet. Porto Alegre, RS: Sulina.

Ryberg, T. (2008). Privacy, power, place and identity - the construction of mixed spaces in an educational context. In: Internet Research 9.0: Rethinking Community, Rethinking Place, Copenhagen, Denmark,

Tairi, K., Mccormack, R., Leihy, P. e Ring, P. (2008). Fairy tales and Elggs: social networking with student rovers in learning commons. In: VALA 14th Biennial Conference and Exhibition, Melbourne, Australia.

Wilson, S.; Beauvoir, P.; Milligan, C.; Sharples, P.; Johnson, M.; Liber, O. (2006) Personal Learning Environments: Challenging the dominant design of educational systems. In: I Joint International Workshop on Professional Learning, Competence Development and Knowledge Management - Lokmol and L3NCD, Heraklion, Outubro 2006. Proceedings... http://hdl.handle.net/1820/727, set. 2012. 